\title{
Developing a Data Driven Approach for Early Detection of SIRS in Pediatric Intensive Care Using Automatically Labeled Training Data
}

\section{Marcel MAST ${ }^{\mathrm{a}, 1}$, Michael MARSCHOLLEK ${ }^{\mathrm{a}}$, ELISE STUDY GROUP*, Thomas JACK $^{\mathrm{b}}$ and Antje WULFF ${ }^{\mathrm{a}}$}

${ }^{a}$ Peter L. Reichertz Institute for Medical Informatics of TU Braunschweig and

Hannover Medical School, Karl-Wiechert-Allee 3, 30625 Hannover, Germany

${ }^{\mathrm{b}}$ Department of Pediatric Cardiology and Intensive Care Medicine, Hannover Medical

School, Carl-Neuberg-Str. 1, 30625 Hannover, Germany

*ELISE STUDY GROUP: Louisa Bode ${ }^{a}$; Marcel Mast ${ }^{a}$; Antje Wulff ${ }^{\text {; }}$ Michael Marschollek $^{a}$; Sven Schamer ${ }^{b}$; Henning Rathert ${ }^{b}$; Thomas Jack ${ }^{b}$; Philipp Beerbaum ${ }^{b}$; Nicole Rübsamen ${ }^{c}$; Julia Böhnke ${ }^{c}$; André Karch ${ }^{c}$; Pronaya Prosun ${ }^{d}$; Lena Wiese ${ }^{d}$; Christian Groszewski-Anders $^{e}$; Andreas Haller ${ }^{e}$, Mathias Slawik $^{e}$; Torsten Frank ${ }^{e}$

${ }^{a}$ Peter L. Reichertz Institute for Medical Informatics of TU Braunschweig and Hannover Medical School, Hannover, Germany

${ }^{\mathrm{b}}$ Department of Pediatric Cardiology and Intensive Care Medicine, Hannover Medical School, Hannover, Germany

${ }^{\mathrm{c}}$ Institute of Epidemiology and Social Medicine, University of Muenster, Muenster, Germany

${ }^{\mathrm{d}}$ Research Group Bioinformatics, Fraunhofer Institute for Toxicology and Experimental Medicine, Hannover, Germany

e medisite GmbH, Hannover, Germany

\begin{abstract}
Critical care can benefit from analyzing data by machine learning approaches for supporting clinical routine and guiding clinical decision-making. Developing data-driven approaches for an early detection of systemic inflammatory response syndrome (SIRS) in patients of pediatric intensive care and exploring the possibility of an approach using training data sets labeled automatically beforehand by knowledge-based approaches rather than clinical experts. Using naïve Bayes classifier and an artificial neuronal network (ANN), trained with real data labeled by (1) domain experts ad (2) a knowledge-based decision support system (CDSS). Accuracies were evaluated by the data set labeled by domain experts using a 10 -fold cross validation. The ANN approach trained with data labeled by domain experts yielded a specificity of 0.9139 and sensitivity of 0.8979 , whereas the approach trained with a data set labeled by a knowledge-based CDSS achieves a specificity of 0.9220 and a sensitivity of 0.8887 . ANN yielded promising results for data-driven detection of pediatric SIRS with real data. Our comparison shows the feasibility of using training data labeled automatically by knowledge-based approaches rather than manually allocated by experts.
\end{abstract}

\footnotetext{
${ }^{1}$ Corresponding Author: Marcel Mast, Peter L. Reichertz Institute for Medical Informatics of TU Braunschweig and Hannover Medical School, Carl-Neuberg-Str. 1, 30625 Hannover, Germany. Email:marcel.mast@plri.de
} 
Keywords. Pediatrics, SIRS, data-driven, machine learning

\section{Introduction}

Medical practice, especially within the pediatric intensive care, is a highly challenging and time pressuring domain. Long-term success of treatment and patient's outcome depend on the timely detection of underlying diseases and an early initiation of a therapy.[1] Nowadays, detection of serious diseases can be supported by analyzing routine clinical data though data-driven applications. Together with the increasing availability of medical data such approaches gain in importance for clinical settings.[2] Hence, data-driven approaches are an a recent considered topic in the field of research. [3, 4] One of these highly challenging diseases within the setting of the pediatric intensive care is the systemic inflammatory response syndrome (SIRS). In 2017, worldwide 2.9 million deaths associated with SIRS were reported for the group of children under five years.[5] To accommodate the differences between adults and children the International Pediatric Sepsis Consensus Conference (IPSCC) has modified the existing adult diagnostic criteria into six age-depending criteria groups for diagnosing SIRS in pediatric patients.[6] According to these criteria SIRS in children manifests when two of the following four criteria, from which one have to be an abnormal temperature or leukocyte count, are met: I) hyper- or hypothermia, II) tachycardia, III) tachy- or bradypnoea, IV) leukocyctosis or leukopenia.[6] The appropriate antimicrobial therapy should be started within an hour of the onset of first symptoms to avoid a two to five times higher mortality.[7] The use of clinical decision-support systems (CDSS) and data-driven approaches can support early detection of SIRS. However, such approaches are rarely used in the medical routine to support clinical decision making. [2-4] In the context of this work, we strive for developing a data-driven approach for the detection of SIRS within the pediatric intensive care. To achieve this goal we aim at implementing two approaches based on (I) a naïve Bayes classifier and (II) an artificial neuronal network (ANN). Both are trained using a ground truth created by domain experts. The manually creation of labeled data as used in this work is a highly time-consuming and challenging task. Therefore, the second objective of this work is evaluating the feasibility of using trainings data labeled automatically by a knowledge-based CDSS rather than using perfect ground truth label allocated manually.[1]

\section{Methods}

In this work we use an existing data set of routine data from the pediatric intensive care unit of the Hannover Medical School. This pseudonymized data set originates from a previously published study.[8] The data set consists of various vital parameters like temperature and blood pressure values, heart and respirations rates as well as laboratory test results and data from medical devices such as cooling blankets, ventilation and pacemaker for each of the included 168 pediatric patients. Laboratory test results include leukocyte, platelet and neutrophil counts as well as INR values derived from the prothrombin time. Every value comes with a specific timestamp documenting its measurement time by which a temporal sequence is ensured. Furthermore, the age of the respective patients is given, which is decisive for a correct pediatric SIRS diagnosis. In addition to the routine data, there is a ground truth describing whether patients suffered 
from SIRS. For the generation of this ground truth two experienced pediatric intensive care physicians manually assessed the patients according to SIRS diagnostic rules defined by the IPSCC.[6] The resulting labeled data is used for training the data-driven algorithms. To improve the quality of the available data the data set has been cleaned and formatted. Furthermore different strategies for missing values were applied.[9] For ANN, missing values were replaced with previous occurred values within a parameterdependent time window. Values that could not be replaced with that imputation strategy were replaced with NULL values. For the naïve Bayes classifier, missing values were ignored. The evaluation of the algorithms comprised splitting the available data set into trainings and test data set and running multiple 10-fold cross validation. Furthermore, the ANN were trained using data automatically labeled by a knowledge-based CDSS and evaluated with the ground truth allocated manually by domain experts.

\section{Results}

The trained algorithms were evaluated individually with the ground truth. The results of multiple 10-fold cross validations for every approach can be found in Table 1. The reported values represent the calculated average from all validation rounds. Folds were generated per patient to avoid splitting related data. The approach based on the naïve Bayes classifier achieves an accuracy from 0.5425 to 0.5683 . Specificity ranges from 0.5848 to 0.6132 and sensitivity from 0.3510 to 0.4230 . The ANN trained on data labeled by clinical experts achieves an accuracy of 0.8975 , ranging from 0.8816 to 0.9174 . The ANN using trainings data labelled by a knowledge-based CDSS achieves an accuracy of 0.8963 , ranging from 0.8693 to 0.9263 . This ANN shows a slightly higher specificity, but a lower sensitivity than the ANN trained with the data labeled by domain experts.

Table 1. Overview of aggregated results of multiple 10 -fold cross validations

\begin{tabular}{|l|c|c|c|}
\hline & Accuracy & Specificity & Sensitivity \\
\hline Naïve Bayes Classifier & 0.5512 & 0.6047 & 0.3894 \\
& {$[0.5425-0.5683]$} & {$[0.5848-0.6132]$} & {$[0.3510-0.4230]$} \\
\hline $\begin{array}{l}\text { ANN trained with data } \\
\text { labeled by domain experts }\end{array}$ & 0.8975 & 0.9139 & 0.8979 \\
\hline $\begin{array}{l}\text { ANN trained with data } \\
\text { labeled by a CDSS }\end{array}$ & $0.8816-0.9174]$ & {$[0.8558-0.9684]$} & {$[0.8650-0.9231]$} \\
\hline
\end{tabular}

The architecture of the used ANN is shown in Table 2.

Table 2. Architecture of the used artificial neuronal network

\begin{tabular}{cccc}
\hline Layer & Type & Number of neurons & Activation function \\
\hline 1 & Input & $8 \times 1$ & tanh \\
2 & Hidden & 6 & softmax \\
\hline
\end{tabular}

\section{Discussion}

With our work, we aimed at examining data-driven approaches for SIRS detection for patients of the pediatric intensive care. Furthermore, we strived to explore the feasibility of using a training data labeled by a knowledge-based CDSS instead of laborious labeled data by clinical experts. Limitations within this work are present in regards of transferability of the approach to other areas and the development and evaluation of the 
used CDSS. Although the naïve Bayes classifier is well known as a solid baseline approach for starting data-driven research [9] our approach based on the naïve Bayes classifier reached a poor accuracy of 0.5512 for a binary classification problem. Even a comparably simpler classifier as ZeroR [10] that just predicts on basis of the majority class would have yielded a higher accuracy of approximately 0.74 for this use case. In contrast, the ANN achieved a promising accuracy. Moreover, literature review revealed that the developed ANN algorithm yielded better results than previously published datadriven approaches for SIRS detection in pediatric patients.[3, 4] The ANN using a data set labeled by a knowledge-based CDSS rather than clinical experts achieved relatively comparable results. This shows the feasibility of using such approaches more often in the future to relieve clinical experts and avoid laborious manual labeling of data sets. However, the development of such CDSS suited for providing label for this use case is a time-consuming task itself that could lead to vast amounts of training data.[1]

\section{References}

[1] Wulff A, Haarbrandt B, Tute E, Marschollek M, Beerbaum P, Jack T. An interoperable clinical decisionsupport system for early detection of SIRS in pediatric intensive care using openEHR. Artificial Intelligence in Medicine. 2018; 89:10-23.

[2] Shortliffe EH, Cimino JJ. Biomedical informatics: Computer applications in health care and biomedicine. 3rd edition. New York, NY: Springer. 2006.

[3] Islam MM, Nasrin T, Walther BA, Wu C-C, Yang H-C, Li Y-C. Prediction of sepsis patients using machine learning approach: A meta-analysis. Computer Methods and Programs in Biomedicine. 2019; 170:1-9.

[4] Wulff A, Montag S, Marschollek M, Jack T. Clinical Decision-Support Systems for Detection of Systemic Inflammatory Response Syndrome, Sepsis, and Septic Shock in Critically Ill Patients: A Systematic Review. Methods of Information in Medicine. 2019.

[5] World Health Organization. Sepsis; 2021 [cited 2021 Aug 24]. Available from: URL: https://www.who.int/news-room/fact-sheets/detail/sepsis.

[6] Goldstein B, Giroir B, Randolph A. International pediatric sepsis consensus conference: definitions for sepsis and organ dysfunction in pediatrics. Pediatric critical care medicine: a journal of the Society of Critical Care Medicine and the World Federation of Pediatric Intensive and Critical Care Societies. 2005; 6(1):2-8.

[7] Scott L. Weiss, Mark J. Peters, Waleed Alhazzani, Michael S. D. Agus, Heidi R. Flori, David P. Inwald et al. Surviving sepsis campaign international guidelines for the management of septic shock and sepsisassociated organ dysfunction in children. Intensive Care Med, Springer. 2020; 46(1):10-67.

[8] Antje Wulff, M. Marschollek. Learning Healthcare Systems in Pediatrics: Cross-Institutional and DataDriven Decision-Support for Intensive Care Environments (CADDIE). Studies in health technology and informatics. 2018; 251: 109-112.

[9] Berthold M, Borgelt C, Höppner F, Klawonn F. Guide to intelligent data analysis: How to intelligently make sense of real data. London: Springer; 2010.

[10] Witten IH, Frank E, Hall MA. Data mining: Practical machine learning tools and techniques. Third edition. Amsterdam: Morgan Kaufmann; 2011. 